\title{
Performance Research on Anti-noise AM Spot Jamming of PRBC-SFM Combined Fuze
}

\author{
Li Gongke ${ }^{1}$, He Shuaitian \\ The Department of Aeronautical Ammunition Engineering \\ The First Aeronautical College of Air Force ${ }^{1}$ \\ Xinyang Normal University ${ }^{2}$ \\ Xinyang, China \\ e-mail: ligongke2010@sina.com ${ }^{1}$
}

\begin{abstract}
This paper gives the research on the anti-noise AM spot jamming performance of pseudo-random binary code phase modulation and sine FM combined fuze based on the criterion of signal to jamming ratio gain. First, the overall signal to jamming ratio gain of its receiver is reduced in detail under the noise AM spot jamming, then, the factors affecting anti-jamming performance are analyzed. The results show that the PRBC-SFM combined fuze has stronger performance of anti-noise AM spot jamming, and its anti-jamming performance can be improved effectively by choosing the parameters of the n-th order Bessel function, and along with decreasing periods and narrowing code width, Doppler frequency's influence on the anti-noise AM spot jamming performance of the PRBC-SFM combined fuze is debased.
\end{abstract}

Keywords-pseudo-random code; sine FM; noise AM jamming; SJR gain; anti-jamming

\section{INTRODUCTION}

In order to antagonize the complicated electromagnetism campaign circumstance and improve the anti-jamming performance of wireless fuze, the development trend of wireless fuze goes towards more complex modulation mode and multiple system nowadays [1-2]. The pseudo-random binary code phase modulation and sine FM (PRBC-SFM ) combined modulation wireless fuze is a new kind of combined fuze, which is gotten in the base of PRBC-SFM by the carrier wave is modulated $0 / \pi$ by the pseudo-random code and exists in the form of fuze transmission signal. This fuze has some good performance, such as the favorable distance identification ability, speed identification ability and low nearby leakage electrical level. Therefore, it is an ideal combined fuze.

It is important for the development and application of this kind of combined fuze to study the anti-jamming performance of the pseudo-random code complex system fuze. For the anti-jamming performance of the PRBC-SFM combined fuze, more documents adopt the method of qualitative analysis, and the quantitative analysis method is less adopted. In this paper, the SJR gain is taken as the reference point, the overall SJR gain of its receiver is reduced in detail under the noise AM spot jamming. And then, the factors affecting anti-jamming performance are analyzed. All the above works propose the theory basis of the parameters optimizing design.

\author{
Tu Youchao \\ College of Physics and Electronics Engineering, Xinyang \\ Normal University \\ Xinyang, China \\ tyc0660@163.com
}

\section{STRUCTURE AND WORK PRINCIPLE}

The PRBC-SFM combined fuze is composed of solidity source, sine modulator, pseudo code producer, $0 / \pi$ phase modulator, frequency mixer, video frequency amplifier, $n$ time frequency multiplier, false-alarm amplifier, correlation and signal processing circuit, which is shown in Fig. 1.

The fuze completes the composite modulation signal transmitting, receiving and signal processing function. The working principle is given below. When works, solid state source generates an RF signal, firstly loads a sinusoidal FM signal, through the pseudo random code PM, and then transmitted by the antenna. The target echo signal is mixed with the FM carrier signal coming from the solid state source, and the intermediate frequency signal is output, after passing the video amplification, which is sent into the selected sideband amplifier. And the sideband signals are chosen and send into the quadratic mixer. After quadratic mix with the corresponding harmonic signal coming from the sine wave modulator, the output difference frequency signal is treated by the CFAR amplifier, put into the correlator with the local delay pseudo random code, the correlator output signal pas the Doppler amplifier, the target information of range and Doppler information is get. The information pass through the signal processing circuit, and the startup signal come into being, and spring the implement.

\section{ThE OVERALl SJR GAIN UNDER THE NOISE AM SPOT JAMMING}

On the basis of the PRBC-SFM combined fuze work principle, in the condition of the noise AM jamming exists, the combined fuze receiver model is shown in Fig. 2.

The combined modulation signal sent by PRBC-SFM combined fuze antenna is (1):

$$
u_{t}(t)=U_{t} S_{p n}(t) \cos \left(\omega_{0} t+m_{f} \sin \omega_{m} t\right)
$$

After the transmitting signal enter the fuze receiver forepart from the channel, the value of the got echo signal can be described as (2): 


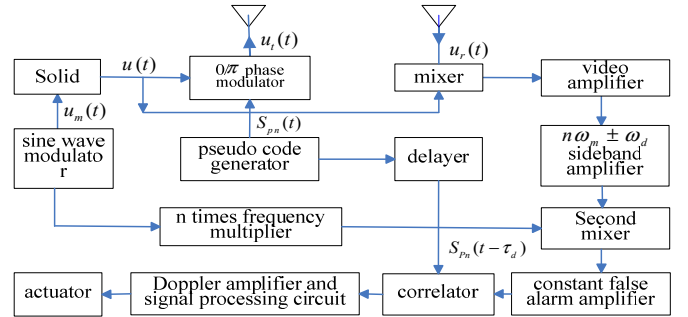

Figure1. Functional block diagram of the PRBC-SFM combined fuze.

$$
u_{r}(t)=\alpha U_{t} S_{p n}(t-\tau) \cos \left[\omega_{0}(t-\tau)+m_{f} \sin \omega_{m}(t-\tau)\right]
$$

Where, $\alpha<1$ is the coefficient concerning with the echo ability of the echo substance, the azimuth, the distance between the echo substance and the missile, $R$. If the echo ability of the echo substance is stronger and the distance is smaller, the value of $\alpha$ will be larger. $\tau=2 R / \mathrm{c}$ is the echo delay time, $\mathrm{c}$ is the velocity of light, $m_{f}=\Delta \omega_{m} / \omega_{m}$ is the frequency modulation index.

The input signal power is

$$
P_{i s}=\frac{1}{2} \alpha^{2} U_{t}^{2}<S_{p n}^{2}(t-\tau)>=\alpha^{2} U_{t}^{2} / 2
$$

If the delay of the noise AM jamming signal reach the fuze receiver is not concerned, the noise AM jamming signal entering the receiver forepart is (4),

$$
J_{r}(t)=\left[U_{0}+n(t)\right] \cos \omega_{j} t
$$

Where, $\omega_{j}$ is the noise jamming carrier frequency, whose value approaches the fuze work frequency. The average value of the noise modulation voltage $n(t)$ is 0 , the square difference $\sigma^{2}$ is Gauss white noise, its power chart density is

$$
G_{n}(f)= \begin{cases}\sigma_{n}^{2} / \Delta F_{n} & 0 \leq f \leq \Delta F_{n} \\ 0 & o . w\end{cases}
$$

Then, the average power of the input jamming signal is

$$
P_{i j 1}=<\left[U_{0}+u_{n}(t)\right]^{2} \cos ^{2} \omega_{j} t>=\frac{1}{2}\left(U_{0}^{2}+\sigma_{n}^{2}\right)
$$

Therefore, the input signal interference ratio of PRBCSFM composite fuze receiver is

$$
S J R_{i}=P_{i s} / P_{i j}=\alpha^{2} U_{t}^{2} /\left(U_{0}^{2}+\sigma_{n}^{2}\right)
$$

Band pass filter (BPF) can be regarded as a band for ideal rectangular filter, to ensure the useful signal $u_{r}(t)$, pass smoothly, $1 / 2 T \gg>m_{f} \omega_{m}$ must be satisfied. With the interference of $f_{j} \approx f_{0}$, the interference signal coming from BPF is an AM signal.

$$
u_{j}(t)=\left[U_{0}+n^{\prime}(t)\right] \cos \omega_{j} t
$$

Where, $n^{\prime}(t)$ is Gauss white noise that the average value is 0 and the square difference is $\sigma_{n}^{2} /\left(2 T \cdot \Delta F_{n}\right)$.

The output of the BPF is

$$
u_{B}(t)=u_{r}(t)+u_{j}(t)
$$

Take FM carrier signal as the vibration signal for the first mixing frequency, that is to say

$$
u_{l 1}(t)=U_{l 1} \cos \left(\omega_{0} t+m_{f} \sin \omega_{m} t\right)
$$

After the signal $u_{b}(t)$ pass mixer, the output difference frequency signal becomes

$$
\begin{aligned}
u_{i 1}(t)= & \frac{1}{2} \alpha U_{t} U_{l 1} S_{p n}(t-\tau) \cos \left[\omega_{0} \tau+2 m_{f} \sin \frac{\omega_{m} \tau}{2} \cos \omega_{m}\left(t-\frac{\tau}{2}\right)\right] \\
& +\frac{1}{2} U_{l 1}\left[U_{0}+n^{\prime}(t)\right]\left[\cos \left(\omega_{0}-\omega_{j}\right) t \cdot \cos \left(m_{f} \sin \omega_{m} t\right)\right. \\
& \left.+\sin \left(\omega_{0}-\omega_{j}\right) t \cdot \sin \left(m_{f} \sin \omega_{m} t\right)\right]
\end{aligned}
$$

With the interfering of collimation form, $\omega_{j} \approx \omega_{0}$.

$$
\begin{aligned}
u_{i 1}(t)= & \frac{1}{2} \alpha_{t} U_{l 1} S_{p n}(t-\tau) \cos \left[\omega_{0} \tau+2 m_{f} \sin \frac{\omega_{m} \tau}{2} \cos \omega_{m}\left(t-\frac{\tau}{2}\right)\right] \\
& +\frac{1}{2} U_{l 1}\left[U_{0}+n^{\prime}(t)\right] \cos \left(m_{f} \sin \omega_{m} t\right)
\end{aligned}
$$

In the following, (12) will be expanded with the first order Bessel function. In order to analyze expediently, let

$$
\begin{aligned}
u_{i 1 u}(t)= & \frac{1}{2} \alpha U_{t} U_{l 1} S_{p n}(t-\tau) \cos \left[\omega_{0} \tau\right. \\
& \left.+2 m_{f} \sin \frac{\omega_{m} \tau}{2} \cos \omega_{m}\left(t-\frac{\tau}{2}\right)\right] \\
u_{i 1 j}(t)= & \frac{1}{2} U_{l 1}\left[U_{0}+n^{\prime}(t)\right]\left[\cos \left(\omega_{0}-\omega_{j}\right) t \cdot \cos \left(m_{f} \sin \omega_{m} t\right)\right. \\
+ & \left.\sin \left(\omega_{0}-\omega_{j}\right) t \cdot \sin \left(m_{f} \sin \omega_{m} t\right)\right] \\
u_{i 1 u}(t)= & \frac{1}{2} \alpha U_{t} U_{l l} S_{p n}(t-\tau)\left\{\cos \omega_{0} \tau \cdot J_{0}(M)\right. \\
& +2 \cos \omega_{0} \tau \cdot \sum_{n=1}^{\infty} J_{2 n}(M)(-1)^{n} \cos \left[2 n \omega_{n}\left(t-\frac{\tau}{2}\right)\right] \\
& \left.+2 \sin \omega_{0} \tau \cdot \sum_{n=1}^{\infty} J_{2 n-1}(M)(-1)^{n} \cos \left[(2 n-1) \omega_{m}\left(t-\frac{\tau}{2}\right)\right]\right\}
\end{aligned}
$$
then

Consider $\omega_{0} \gg \omega_{m}, n \omega_{m} \tau$ and $(2 n-1) \omega_{m} \tau / 2$ can be omitted,

$$
\begin{aligned}
& u_{i l u}(t)=\frac{1}{2} \alpha U_{t} U_{l 1} S_{p n}(t-\tau)\left\{J_{0}(M) \cdot \cos \omega_{0}\right. \\
& +\sum_{n=1}^{\infty}(-1)^{n} J_{2 n}(M)\left[\cos \left(\omega_{0} \tau+2 n \omega_{m} t\right)+\cos \left(\omega_{0} \tau-2 n \omega_{m} t\right)\right] \\
& \left.+\sum_{n=1}^{\infty}(-1)^{n} J_{2 n-1}(M)\left[\cos \left(\omega_{0} \tau+(2 n-1) \omega_{m} t\right)+\cos \left(\omega_{0} \tau-(2 n-1) \omega_{m} t\right)\right]\right\}
\end{aligned}
$$

Where, $M=2 m_{f} \sin \omega_{m} \tau / 2$.

Considering the Doppler effect, let the adjacency speed be $v_{R}$, then,

By (16) and (17),

$$
\tau=\tau_{0}-2 v_{R} t / c, \omega_{0} \tau=\omega_{0} \tau_{0}-\omega_{d} t
$$

$$
\begin{aligned}
u_{i 1 u}(t)= & \frac{1}{2} \alpha U_{t} U_{l 1} S_{p n}(t-\tau)\left\{J_{0}(M) \cdot \cos \left(\omega_{d} t-\omega_{0} \tau_{0}\right)\right. \\
& -J_{1}(M)\left[\sin \left(\left(\omega_{m}-\omega_{d}\right) t+\omega_{0} \tau_{0}\right)-\sin \left(\left(\omega_{m}+\omega_{d}\right) t-\omega_{0} \tau_{0}\right)\right] \\
& -J_{2}(M)\left[\cos \left(\left(2 \omega_{m}-\omega_{d}\right) t+\omega_{0} \tau_{0}\right)+\cos \left(\left(2 \omega_{m}+\omega_{d}\right) t-\omega_{0} \tau_{0}\right)\right] \\
& +J_{3}(M)\left[\sin \left(\left(3 \omega_{m}-\omega_{d}\right) t+\omega_{0} \tau_{0}\right)-\sin \left(\left(3 \omega_{m}+\omega_{d}\right) t-\omega_{0} \tau_{0}\right)\right] \\
& +J_{4}(M)\left[\cos \left(\left(4 \omega_{m}-\omega_{d}\right) t+\omega_{0} \tau_{0}\right)\right. \\
& \left.\left.+\cos \left(\left(4 \omega_{m}+\omega_{d}\right) t-\omega_{0} \tau_{0}\right)\right]+\cdots\right\}
\end{aligned}
$$

get

Expand (12) with the first order Bessel function, will 


$$
u_{i 1 j}(t)=\frac{1}{2} U_{l 1}\left[U_{0}+n^{\prime}(t)\right] \cdot\left[J_{0}(M)+\sum_{n=1}^{\infty} 2 J_{2 n}\left(m_{f}\right) \cos 2 n \omega_{m} t\right]
$$

$u_{i 1}(t)$ goes through the sideband amplifier which the bandwidth is $\left(n \omega_{m}-B / 2, n \omega_{m}+B / 2\right)$, in order to ensure only the $n$ power sideband to be chosen, the bandwidth, $B$, should be $2 \omega_{d}<B<2\left(\omega_{m}-\omega_{d}\right)$. When $\mathrm{n}$ is even, the chosen $\mathrm{n}$ power sideband signal is

$$
\begin{aligned}
u_{n}(t) & =\frac{1}{2} k \alpha U_{t} U_{l 1} S_{p n}(t-\tau) J_{n}(M)\left\{\cos \left[\left(n \omega_{m}-\omega_{d}\right) t+\omega_{0} \tau_{0}\right]\right. \\
& \left.+\cos \left[\left(n \omega_{m}+\omega_{d}\right) t-\omega_{0} \tau_{0}\right]\right\}+k U_{l 1}\left[U_{0}+n^{\prime \prime}(t)\right] . J_{n}\left(m_{f}\right) \cos n \omega_{m} t
\end{aligned}
$$

In (20), $k$ denotes the sideband amplifier gain, $\omega_{0} \tau_{0}$ is the initial phase of $n$ powers harmonic with no useful information, therefore, in the following, which will be omitted. $n^{\prime \prime}(t)$ is Gauss noise with average value zero and square difference $B \sigma_{n}{ }^{2} /\left(2 \Delta F_{n}\right)$, the power chart density is

$$
G_{n}^{\prime \prime}(f)= \begin{cases}\sigma_{n}{ }^{2} / \Delta F_{n} & 0 \leq f \leq \frac{B}{2} \\ 0 & \text { o.w }\end{cases}
$$

The autocorrelation function is

$$
R_{n^{\prime \prime}}\left(\tau^{\prime}\right)=\frac{B \sigma_{n}^{2}}{2 \Delta F_{n}} S a\left(\frac{\pi B \tau^{\prime}}{2}\right) e^{\frac{j \pi B \tau^{\prime}}{2}}
$$

Let the output signal of the $\mathrm{n}$ times doubler is

$$
u_{l 2}(t)=U_{l 2} \cos n \omega_{m} t
$$

$u_{n}(t)$ enter the second mixer for mixing processing, the output of the difference frequency signal is

$$
\begin{aligned}
u_{i 2}(t)= & \frac{1}{2} k U_{l 1} U_{l 2}\left\{\alpha U_{t} J_{n}(M) S_{p n}(t-\tau) \cos \omega_{d} t\right. \\
& \left.+J\left(m_{f}\right)\left[U_{0}+u^{\prime \prime}(t)\right]\right\}
\end{aligned}
$$

Processed by the constant false alarm amplifier, the gotten output signal is

$$
\begin{aligned}
u_{o}(t) & =S_{p n}(t-\tau) \cos \omega_{d} t \\
& +J_{n}\left(m_{f}\right)\left[U_{0}+u_{n}^{\prime \prime}(t)\right] / \alpha U_{t} J_{n}(M)
\end{aligned}
$$

Let the local delay code is $S_{p n}\left(t-\tau_{d}\right)$, where $\tau_{d}$ determine the best distance of the combined fuze. After relevant processing, the output $R_{u S}\left(\tau_{d}\right)$ is given, then

$$
R_{u S}\left(\tau_{d}\right)=\frac{1}{p T} \int_{0}^{p T} u_{o}(t) S_{p n}\left(t-\tau_{d}\right) d t=R_{S S}\left(\tau_{d}-\tau\right)+R_{j S}\left(\tau_{d}\right)
$$

Where, $R_{S S}\left(\tau_{d^{-}} \tau\right)$ is the useful signal coming from the correlator, $R_{j S}\left(\tau_{d}\right)$ is disturbance. When $\tau_{d=\tau} \tau$, the maximum value of the useful signal coming from the correlator is

$$
R_{S S}\left(\tau_{d}-\tau\right)_{\max }=\sin c\left(p T \omega_{d}\right)
$$

The maximum peak power of the correlator output is

$$
\left(P_{o s}\right)_{\max }=\sin c^{2}\left(p T_{m} \omega_{d}\right)
$$

The average power of the correlator output disturbance is

$$
\begin{aligned}
& P_{o j}=<R_{j S}^{2}\left(\tau_{d}\right)>=\frac{1}{(p T)^{2}} \int_{0}^{p T} \int_{0}^{p T}\left[\frac{J_{n}\left(m_{f}\right)}{\alpha U_{t} J_{n}(M)}\right]^{2} . \\
& {\left[U_{0}^{2}+<u_{n}^{\prime \prime}\left(t_{1}\right) u_{n}^{\prime \prime}\left(t_{2}\right)>\right] S_{p n}\left(t_{1}-\tau_{d}\right) S_{p n}\left(t_{2}-\tau_{d}\right) d t_{1} d t_{2} } \\
&=\left[J_{n}\left(m_{f}\right) / \alpha U_{t} J_{n}(M) p T\right]^{2 T} \int_{0}^{p T} \int_{0}^{p T}\left[U_{0}^{2}+R_{n^{\prime \prime}}\left(t_{1}-t_{2}\right)\right] . \\
& S_{p n}\left(t_{1}-\tau_{d}\right) S_{p n}\left(t_{2}-\tau_{d}\right) d t_{1} d t_{2}^{0}
\end{aligned}
$$

When $t_{1}=t_{2}, P_{o j}$ takes maximum, then

$$
\left(P_{o j}\right)_{\max }=\left[U_{0}^{2}+\frac{B \sigma_{n}^{2}}{2 \Delta F_{n}}\right] \cdot\left[J_{n}\left(m_{f}\right) / \alpha U_{t} J_{n}(M)\right]^{2}
$$

Signal to jamming ratio of the maximum peak coming from the correlator is

$$
S J R_{o}=\left[\frac{\alpha U_{t} J_{n}(M)}{J_{n}\left(m_{f}\right)}\right]^{2} \cdot \frac{\sin c^{2}\left(p T \omega_{d}\right)}{U_{0}^{2}+B \sigma_{n}^{2} / 2 \Delta F_{n}}
$$

The total SJR gains of receiver is

$$
G=\frac{S J R_{o}}{S J R_{i}}=\left[\frac{J_{n}(M)}{J_{n}\left(m_{f}\right)}\right]^{2} \cdot \frac{\left(1+m_{A E}^{2}\right) \cdot \sin c^{2}\left(p T \omega_{d}\right)}{1+B m_{A E}^{2} / 2 \Delta F_{n}}
$$

Where, $m_{A E}=\sigma_{n} / U_{0}$ is the effective modulation coefficient.

\section{PERFormance ANAlysis of ANTI-NOISE AM SpOT} JAMMING OF COMBINED FUZE

\section{A. Detected Frequency of the Pseudo-Random Code} System Fuze and Interfered with Spot Jamming

Supposing that the working frequency of the pseudorandom code system fuze is detected and interfered with spot jamming, their frequency should meet the following conditions.

$$
f_{j} \approx f_{0}, \Delta f_{j}=(2 \sim 5) \Delta f_{r}
$$

In this paper, $\Delta f_{j}=2 \Delta F_{n}, \Delta f_{r}=1 / T$, and $2 \omega_{d}<B<2\left(\omega_{m^{-}}\right.$ $\left.\omega_{d}\right), 1 / 2 T \gg m_{f} \omega_{m}, m_{f} \gg>1$. So, considering these conditions, can conclude $\Delta F_{n} \gg B$.

According to the reference [5], $m_{A E}=m_{A} / K_{C}, m_{A}$ is the maximum modulation coefficient, $m_{A} \leq 1, K_{C}$ is the noise peak factor. To improve the noise quality and gain biggish $m_{A E}$, $K_{C}=1.4 \sim 2$ [6]. So, $m_{A E} \leq 1 / K_{C}=1 / 2 \sim 1 / 1.4$. Set $m=(1+$ $\left.m_{A E}^{2}\right) /\left(1+B m_{A E}^{2} / 2 \Delta F_{n}\right), \Delta F_{n}=50 \mathrm{MHz}, B=400 \mathrm{KHz}$,

Then, the relationship between $m$ and $m_{A E}$ is showed in TABLE I.

TABLE I. RELATIONSHIP BETWEEN $m$ AND $m_{A E}$

\begin{tabular}{|c|c|l|l|l|l|l|l|}
\hline $\mathbf{m}_{\mathbf{A E}}$ & $\mathbf{1} / \mathbf{2}$ & $\mathbf{1} / \mathbf{1 . 9}$ & $\mathbf{1} / \mathbf{1 . 8}$ & $\mathbf{1} / \mathbf{1 . 7}$ & $\mathbf{1} / \mathbf{1 . 6}$ & $\mathbf{1} / \mathbf{1 . 5}$ & $\mathbf{1 / 1 . 4}$ \\
\hline $\mathrm{m}$ & 1.25 & 1.28 & 1.31 & 1.34 & 1.39 & 1.44 & 1.51 \\
\hline
\end{tabular}

Seen from TABLE I, should have

$$
G \approx\left[J_{n}(M) / J_{n}\left(m_{f}\right)\right]^{2} \cdot \sin c^{2}\left(p T \omega_{d}\right)
$$

Therefore, the overall SJR gain of the PRBC-SFM combined fuze has nothing to do with the carrier amplitude voltage $U_{0}$ and the modulation noise $n(t)$. It proves that the combined fuze has stronger performance of anti-noise AM jamming.

\section{B. Selection Harmonic Order to Decrease the Noise}

To decrease noise, harmonic order of the sideband amplifier should be greater than 3 [7]. Set $J=J_{n}(M) / J_{n}\left(m_{f}\right)$ and $\mathrm{n} \geq 3$. Because the selected harmonic order in this paper is even-order harmonic, variation curves of $J$ following $m_{f}$ when $n=4,8$ is shown in Fig. 3 . 


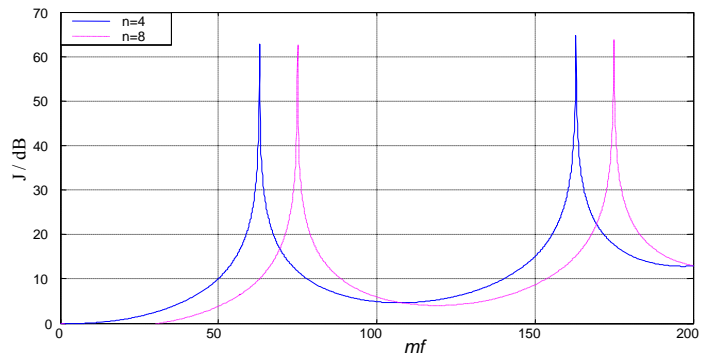

Figure3. Variation curve of $J$ following $m_{f}$

Seen from Fig. 3, when $n$ is fixed, $J$ increases with $m_{f}$ rising, and $J$ gets its maximum when $m_{f}$ attains a certain value, and $J$ will diminish contrarily if $m_{f}$ sequentially increases. With $n$ increase, the $J \sim m_{f}$ curve shifts to right, which indicates that to keep $J$ a constant value, $m_{f}$ must increase. The bandwidth of BPF must meet the conditions of $1 / 2 T>>m_{f} \omega_{m}$, the width of the code element cannot be set too narrow and $m_{f}$ should not be set too high due to the influence of device performance nowadays.

Therefore, design of the combined fuze reasonable parameter $n, m_{f}, J$ should be fully considered.

\section{Variation of SJR gain with Doppler Frequency}

On the basis of formula (34), Fig. 4 shows the variation curve of the overall SJR gain of PRBC-SFM combined fuze following Doppler frequency under the noise AM spot jamming $\left(f_{0}=f_{j}\right)$.
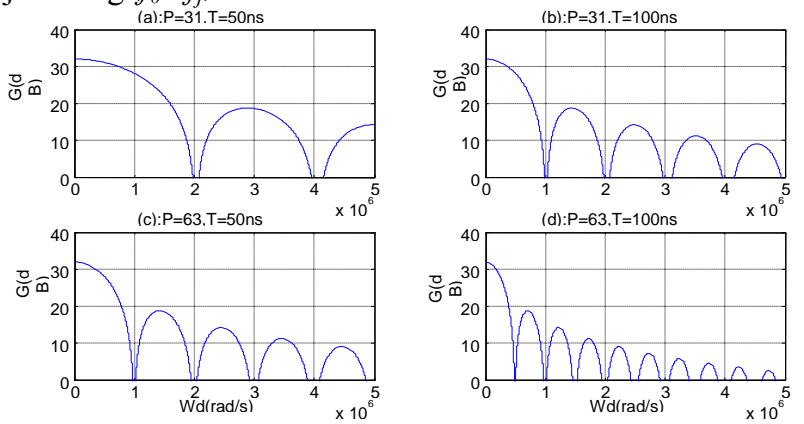

It can be concluded that the period of the pseudo code sequence much longer and the code width much wider, the curve descent speed faster, as $p$ and $T$ are set, the overall SJR gain of the fuze receiver $G$ generally declines with $\omega_{d}$ increase, the distance mensuration of the combined fuze appear fuzzy as $\omega_{d}=n \pi /(p T) n=1,2 \ldots$.

\section{CONCLUSIONS}

The overall SJR gain of the receiver correlator of the PRBC-SFM combined fuze is deduced in detail under the noise AM spot jamming, the factors influencing the antijamming performance of the fuze are analyzed. The results show that the PRBC-SFM combined fuze has stronger performance of anti-noise AM spot jamming, and its antijamming performance can be improved effectively by choosing the parameters of $n$ and $m_{f}$ in the $n$-th order Bessel function. Increasing of periods and code width, the combined fuze noise AM anti-jamming performance is influenced strongly by the Doppler frequency.

\section{REFERENCES}

[1] John G. Rawcliffe, David E. Patrick, John Anderson. Proximity fuze. U.S.. 6834591, December 2004.

[2] Yasuo Naqazumi. Random pulse type radar apparatus. U.S.. 6381261, April 2002.

[3] Xiong Gang, Zhao Hui-chang, Yang Xiao-niu. "Pseudo-random binary- phase coded and sine frequency modulated pulse train fuze," Modern Radar, vol. 29, no. 2, 2007, pp.12-16.

[4] Lin Xiang-pin, Radar countermeasure principle, Xi'an, Northwest Institute of Telecommunication Engineering Publishing House, 1985, pp.248-262.

[5] Zhao Guo-qing, Radar countermeasure principle, Xi'an, Xidian University Publishing House, 2003, pp.124-141.

[6] Han Pei-yao, Radar anti-jamming technology. Beijing. National Defense Industry Press, 1980, pp.66-74.

[7] Cui Zhan-zhong, Song Shi-he, Proximity fuze principle, Beijing, Beijing Institute of Technology Press, 1998, pp.83-84.

Figure4. Variation curve of $G$ following $\omega_{d}$.

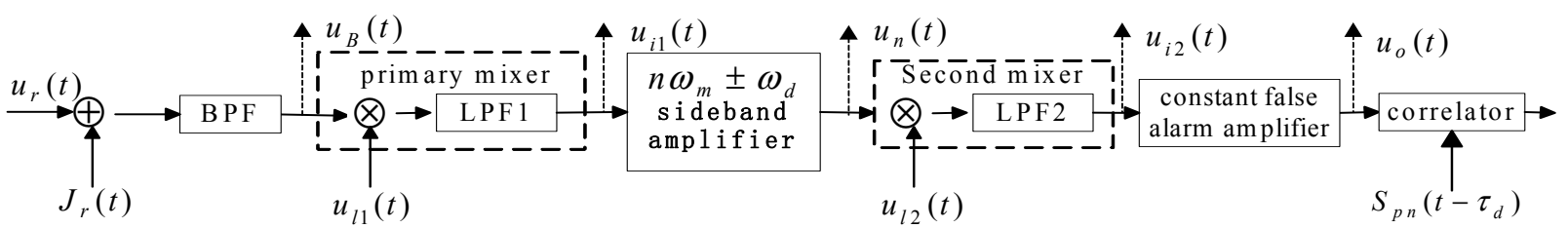

Figure2. Receiver model of the PRBC-SFM combined fuze. 